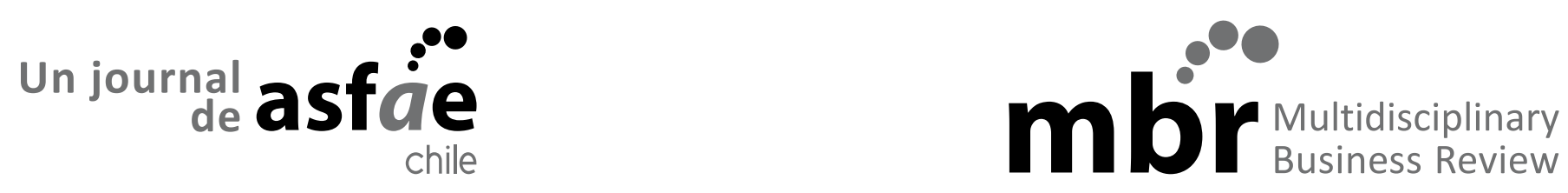

\title{
AUTOESTIMA Y MOTIVACIÓN EN LA COMUNIDAD ESCOLAR, ¿CÓMO INCIDEN EN EL INGRESO A LA EDUCACIÓN SUPERIOR?
}

\author{
SELF-ESTEEM AND MOTIVATION IN THE SCHOOL COMMUNITY, HOW DO \\ THEY AFFECT ADMISSION TO HIGHER EDUCATION?
}

\author{
Elías Karmach-Sánchez ${ }^{\mathrm{a}}$ Carlos Delgado-Fuentealba ${ }^{\mathrm{b}} \cdot$ Paola Zerega-Tallia $^{\mathrm{c}} \cdot$ Mauricio López-Muñoz $^{\mathrm{d}}$ \\ Clasificación: Trabajo empírico-investigación \\ Recibido: 30 -diciembre- 2020 / Revisado: 12 -marzo- 2021 / Aceptado: 16 -marzo- 2021
}

\section{Resumen}

La investigación buscó determinar si existe un efecto del grado de autoestima y motivación del establecimiento escolar de egreso de un estudiante en su ingreso a una universidad versus un instituto técnico profesional en Chile. La delimitación del objeto de estudio se circunscribe a alumnos matriculados en los años 2018 y 2019, en entidades de la Corporación Universidad de Concepción, esto es, Universidad de Concepción (UdeC) e Instituto Profesional Virginio Gómez (IPVG), en la región de Ñuble. La cantidad de observaciones equivale a 2055 alumnos. Desde un punto de vista econométrico, estimamos los factores que inciden en la probabilidad de que un estudiante haya continuado sus estudios en una universidad (UNIV) en vez de un instituto profesional (IP). Utilizamos un modelo Probit binario, puesto que la variable dependiente puede adoptar dos valores discretos (cero o la unidad). Medimos el grado de autoestima y motivación por medio de su respectivo Indicador de Desarrollo Personal y Social determinado por la Agencia de Indicadores de Calidad del Ministerio de Educación. Además, incorporamos variables de control, tales como características personales del estudiante y características del establecimiento de enseñanza media. Los resultados obtenidos revelan que los estudiantes que egresan de establecimientos educacionales secundarios con mayor autoestima y motivación tienen mayor probabilidad de ingresar a la UdeC que al IPVG. Si bien el sistema de educación superior ha avanzado en cobertura, debe avanzar también en el desarrollo de políticas públicas que profundicen en la calidad y contemplen la potenciación de los factores de formación emocional como la autoestima y la motivación.

Palabras clave: motivación, autoestima, educación superior, universidades, institutos profesionales.

\begin{abstract}
The research sought to determine if there is an effect of the degree of self-esteem and motivation of a student's graduation school establishment in his entry to a university versus a professional technical institute in Chile. The object of study was delimited to students enrolled in entities of Corporación Universidad de Concepción, that is, Universidad de Concepción (UdeC) and Instituto Profesional Virginio Gómez (IPVG), in Ñuble region, corresponding to the years 2018 and 2019. The number of observations equals 2055 students. From an econometric point of view, we estimate the factors that influence the probability that a student has continued his studies at a university (UNIV) instead of a professional institute (IP). We use a binary probit model, since the dependent variable can take two discrete values (zero or unity). We measure the degree of self-esteem and motivation through their respective Personal and Social Development Indicator determined by the Quality Indicators Agency of the Ministry of Education. In addition,

\footnotetext{
Escuela de Administración y Negocios, Universidad de Concepción, Campus Chillán. Correo electrónico: ekarmach@udec.cl Escuela de Administración y Negocios, Universidad de Concepción, Campus Chillán. Correo electrónico: cadelgado@udec.cl Escuela de Administración y Negocios, Universidad de Concepción, Campus Chillán. Correo electrónico: paolazerega@udec.cl
} Instituto Profesional Virginio Gómez, Sede Chillán. Correo electrónico: mlopez@virginiogomez.cl
\end{abstract}


we incorporate control variables such as: personal characteristics of the student and characteristics of the secondary school. The results obtained reveal that students who graduate from secondary educational establishments with higher self-esteem and motivation are more likely to enter the UdeC than the IPVG. Although the higher education system has made progress in coverage, it must advance in developing public policies that deepen quality, contemplating enhancing emotional formation factors such as self-esteem and motivation.

Keywords: Motivation, self-esteem, higher education, universities, professional institutes.

\section{Introducción}

En 2007, la Unesco afirmó que la educación es un medio pleno para el desarrollo del ser humano y su fortalecimiento como persona; es decir, más que servir al Estado o a la industria, debe ser utilizada para desarrollar personas en un ámbito tanto espiritual como cultural, con el fin de contribuir al desarrollo de la sociedad a través del conocimiento (Sánchez, 2011). El desarrollo social solo es relevante si progresan todos los miembros de la sociedad por igual (Llanos et al., 2018).

Durante las últimas dos décadas, Chile ha demostrado ser una de las economías más sólidas de Latinoamérica, gracias al buen desempeño de sus instituciones y a su estabilidad macroeconómica; sin embargo, también se ha revelado como uno de los países con mayor desigualdad en el mundo y se posiciona en el segundo lugar de la clasificación de desigualdad dentro de la Organización para la Cooperación y el Desarrollo Económico (OCDE, 2015). Según el coeficiente de Gini, los ingresos del 10\% más rico son 26 veces más altos que los del $10 \%$ más pobre. Del problema en cuestión se desprenden dos factores clave: la educación secundaria y la superior. Ambas se muestran como medios para alcanzar el desarrollo personal, facilitar el acceso al mundo laboral y establecer una mejor movilidad social (Blanco y Cusato, 2004).

El acceso va más allá de los sistemas de admisión, los procesos de selección universitaria mediante Prueba de Selección Universitaria (PSU), las Notas de Enseñanza Media (NEM) y la actualmente incorporada clasificación. Es más bien el resultado de una experiencia educativa, que según World Data on Education de la Unesco (20102011), se compone de doce años obligatorios, donde la educación preprimaria no es obligatoria a diferencia de los niveles de educación primaria de ocho años y educación secundaria de cuatro años.

Como señala la investigación realizada por Rodríguez (2012), la educación recibida en los niveles primario y secundario está determinada por la condición social y económica de las personas, lo que repercute en los resultados de procesos de selección universitaria. Con base en los datos entregados por el Departa- mento de Evaluación, Medición y Registro Educacional (Demre) de la Universidad de Chile, se establece que las condiciones de origen de los estudiantes pueden ser más determinantes que sus talentos para ingresar y elegir la institución de Educación Superior (Llanos et al., 2018).

En el país, un tercio de los estudiantes de tercero y cuarto medio eligen la modalidad de Educación Media Técnico-Profesional (EMTP). Esta proporción es el doble de la de otros países de la región y cercana al promedio de países de la OCDE. Además, un $38 \%$ de la matrícula de primer año de educación superior corresponde a programas de la modalidad de Educación Superior Técnico Profesional, en un sistema en el cual la tasa neta de participación en educación superior es del 70.7\%. Sin embargo, la educación técnico profesional ha sido la opción para jóvenes de menores recursos, y es una alternativa valorada por familias de bajos recursos. El nivel socioeconómico es el determinante principal en la elección de la modalidad de EMTP, pues ejerce un papel incluso más importante que el desempeño académico (Arias et al., 2015).

El perfil de ingreso de los estudiantes a la educación superior se presume como un determinante con alto factor de impacto en el rendimiento académico y la calidad profesional adquirida por una persona que ingresa a la educación terciaria ya sea técnica, mediante Centros de Formación Técnica o Institutos Profesionales; o bien universitaria, mediante universidades tradicionales, públicas o subvencionadas, y privadas. En el caso de las universidades chilenas, los estudiantes que ingresan y se matriculan en primer año en una casa de estudio, independiente del tipo de institución que se trate, poseen arraigado un conjunto de características propias y de su entorno que determinan sus perfiles de ingreso (Llanos et. al, 2018).

En el caso de Ñuble, los números son los siguientes: de un total de 13650 estudiantes matriculados en educación superior, el $57.8 \%$ optó por carreras universitarias; el $23.6 \%$ prefirió carreras de Institutos Técnico Profesionales (IP) y el $18.6 \%$ eligió Centros de Formación Técnica (CFT) (Barómetro del aseguramiento de la calidad de la educación superior, $\left.\mathrm{N}^{\circ} 4,2019\right)$. 


\section{Revisión de literatura}

El papel del Estado en materia de políticas públicas relativas a la educación, resurgió en los años ochenta con dos reformas educacionales que coexisten hasta hoy.

Ambas ocurridas en el último cuarto del siglo XX, coinciden, además, con iguales períodos gubernamentales cruciales en Chile. El primer momento corresponde al régimen que por más de dieciséis años gobernó con poderes "extraordinarios" el país, y el siguiente tiene que ver con el retorno del régimen democrático, desde principios de los años noventa, expresado en coaliciones de partidos a la fecha. Desde ahí se puede desprender que las políticas públicas se habrían limitado a una implementación administrativa, de operación, de financiamiento, de gestión, de regulación y de currículum, con carencia del ámbito actitudinal.

En ese sentido, la coherencia involucra al menos tres elementos básicos: a) el nivel de estructuralidad de las políticas educativas, es decir, hasta qué punto dichas políticas determinan el funcionamiento del sistema en su conjunto; b) el contexto interno de cada establecimiento, entendido como el conjunto de capacidades profesionales e institucionales existentes; $\mathrm{y} \mathrm{c}$ ) el entorno o contexto externo, tal como las condiciones socioeconómicas de la comunidad escolar y los vínculos de apoyo o presión desde los niveles de liderazgo intermedio (Aziz, 2018).

Estas características comprenden antecedentes familiares, tales como el nivel socioeconómico y cultural de la familia, así como los valores que esta sustenta, junto con los atributos personales y de la experiencia académica preuniversitaria. Dichos rasgos distintivos se combinan para influir sobre el compromiso inicial con la institución, así como en la consecución de su meta que es la graduación o titulación (Himmel, 2002).

En este marco, las aspiraciones individuales responden a percepciones compartidas por los miembros de un grupo o clase social y orientan el tipo de elección que les resulta posible. Son evaluaciones subjetivas acerca de las posibilidades de movilidad social y de construcción de una trayectoria personal, pero representan, al mismo tiempo, las probabilidades objetivas de futuro de quienes las construyen. No son análisis racionales, pero constituyen la forma en que los jóvenes de diversas clases sociales construyen elecciones razonables en función del capital cultural del que disponen (Sepúlveda y Valdebenito, 2014).

\section{Autoestima y motivación}

¿Existe alguna relevancia en estudiar la competencia desarrollo de un plan de vida personal o proyecto de vida? La educación es un factor determinante en la con- solidación de aprendizajes que favorezcan la formación integral de estudiantes, tanto en el aprender a hacer como en el aprender a ser. El proyecto de vida de una persona es un quehacer que se realiza de manera constante, día con día, por lo que se requieren de habilidades y conductas positivas tendientes a su concreción (Lomelí, López y Valenzuela, 2016).

Por su parte, D'Angelo (1986) menciona que en la adolescencia el proyecto de vida de jóvenes tiene especial importancia, debido a que se encuentra en estado de elaboración casi total, de tal manera que los sistemas de educación deben contribuir al aprendizaje de las diferentes ciencias y al desarrollo integral de los individuos de acuerdo con sus características personales.

Por lo tanto, es importante conocer los tipos de obstáculos con los que tropiezan las generaciones jóvenes y de qué forma influyen como determinantes de sus logros. También resulta significativo determinar qué factores en la realización o fijación de metas son externos, es decir, relacionados con la familia o con el entorno educativo. ¿Cómo se motivan y de qué manera influyen sus valores y la autoestima para superar obstáculos y seguir adelante con la realización de un proyecto de vida con éxito? Estas son interrogantes que se plantean tanto en el ámbito educativo como en el familiar (Lomelí, López y Valenzuela, 2016).

Entonces resulta relevante incorporar el concepto de proyecto de vida, entendido como el plan futuro que cada persona piensa para sí misma. ¿Qué tan condicionado puede estar un proyecto de vida dado lo anterior? Para Castañeda (2011), trazar un plan de vida ayudaría a los grupos de jóvenes a visualizar un futuro de manera más objetiva y a hacer un compromiso con ellos mismos, además sería útil para aclarar los pasos que deben seguir con el fin de llegar a sus metas. El mismo autor menciona a la autoestima y al elemento familiar, social y económico como aspectos importantes para la realización de un proyecto de vida en los jóvenes. Allí mismo, destaca a la autoestima como necesaria para poder triunfar en la vida, pues menciona que no se puede concebir una persona exitosa si no se cuida ni se quiere.

En línea con lo anterior, la Agencia de Calidad de la Educación, dependiente del Ministerio de Educación de Chile, se centra en los conceptos de autoestima académica y motivación escolar, y los define de la siguiente manera:

Autopercepción y autovaloración académica: incluye tanto las percepciones de los estudiantes frente a sus aptitudes, habilidades y posibilidades de superarse, como la valoración que hacen sobre sus atributos y habilidades en el ámbito académico. 
Motivación escolar: incluye las percepciones de los estudiantes respecto de su interés y disposición al aprendizaje, sus expectativas académicas y motivación al logro, y sus actitudes frente a las dificultades y la frustración en el estudio.

En este sentido, la Agencia de Calidad de la Educación, en su cuestionario Informe Técnico 2017: Indicadores de Desarrollo Personal y Social (IDPS) — que constituye una de las bases de datos de la presente investigaciónconsideró un solo indicador la motivación académica y la motivación al logro, debido a que ambas son variables directamente relacionadas con la autoestima.

Así, Abouserie (1995) y Awan, Noureen y Naz (2011) entregan evidencia sobre la alta correlación entre el autoconcepto y la motivación al logro, demostrando que aquellos estudiantes que tienen un alto concepto académico tienden a presentar una mejor disposición al estudio; metas y expectativas académicas más altas; y un mayor deseo de obtener resultados en este ámbito. A su vez, un número importante de investigaciones muestra la estrecha relación que existe entre el autoconcepto y la motivación académica y el rendimiento académico como Green, 2012; Awan, Noureen y Naz, 2011; Barker, McInerney y Dowson, 2003; y, para otros, la autoestima académica considera variables tales como motivación académica, compromiso, objetivos, valores y autoeficacia que presenta el estudiante en el ámbito escolar (Arnold y Doctoroff, 2003).

Los rasgos de autoestima y motivación, por ejemplo, indican que la primera tiene un carácter multidimensional. Para efectos de esta investigación, se ha circunscrito a la autoestima de tipo académico debido a que es esta esfera en la que el establecimiento educacional tendría mayor influencia y, por lo mismo, una mayor responsabilidad respecto del desarrollo de sus estudiantes.

En consecuencia, una buena autoestima potencia el desarrollo de otros aspectos de la vida de los estudiantes, además, es un factor protector frente a eventos negativos y conductas antisociales, incide en una adaptación flexible y adecuada a las nuevas experiencias y actitudes positivas hacia el entorno. En cambio, una baja autoestima se correlaciona con diversos problemas de personalidad y sociales, tales como trastornos conductuales, depresión, ausentismo escolar, entre otros (Arón y Milicic, 2004; Mujis y Reynolds, 2005.

En el ámbito motivacional, los problemas de personalidad y sociales también se asocian con estudiantes que tienen una baja motivación escolar (Conley y Karabenick, 2006; Green, 2012). Así, la teoría de orientación al logro de metas (Elliot McGregor, 2001) señala que cuando los estudiantes se involucran en las tareas académicas, establecen diversos objetivos personales que pueden influir directamente en sus resultados académicos. Los estudiantes con motivación al logro son más eficaces que otros estudiantes, cumplen con un cierto nivel de excelencia y trabajan duro para alcanzarlo (Awan, Noureen y Naz, 2011).

Por otra parte, la motivación se puede analizar en dos subconstrucciones, basadas en las razones u objetivos que dan lugar a una acción. La distinción más básica es entre la motivación intrínseca, que se refiere a hacer algo porque es intrínsecamente interesante o agradable, y la motivación extrínseca, que se refiere a hacer algo porque conduce a un resultado separable (Ryan y Deci, 2000). En tanto, la motivación extrínseca puede incluir recompensas o incentivos contingentes; es decir, la recompensa depende de la realización satisfactoria de una tarea (Bénabou y Tirole, 2003).

El sistema escolar con su énfasis en las calificaciones y la confirmación externa de parte de los profesores es un ejemplo común de motivación extrínseca y es algo fundamental para tener en consideración, especialmente porque investigaciones muestran que la motivación intrínseca, más que la extrínseca, es fundamental para el aprendizaje y la formación de personas interesadas de por vida en el estudio (Irvine, 2018).

Es ahí donde el entorno educativo es un contexto en que los profesores les otorgan importancia a los logros académicos. Los establecimientos educacionales pueden verse como una gran oportunidad para fomentar competencias adicionales que tendrán un impacto significativo en la vida de los estudiantes, especialmente entre aquellos provenientes de familias más vulnerables. Se ha comprobado que el estudiante puede revertir una percepción negativa de sí mismo mediante interacciones positivas en el contexto escolar, por ello, el colegio puede constituirse como una experiencia compensatoria y remedial (Busso et al., 2012; García y Musitu, 1993).

Finalmente, para complementar lo anterior, el proyecto personal que construyen los jóvenes estudiantes refleja el interés generalizado por ingresar a un centro de educación superior previo al inicio de la vida laboral, lo que ratifica el carácter transversal de la aspiración a una calificación de carácter profesional postsecundaria. Esta tendencia no encuentra diferencias de género y solo se presentan distinciones marginales por nivel socioeconómico y modalidad de estudios cursados en la enseñanza media (Sepúlveda y Valdebenito, 2014).

\section{Métodos de investigación}

A fin de testear nuestra hipótesis, consistente en un posible efecto del grado de autoestima y motivación que envuelven los establecimientos de enseñanza media 
sobre la decisión del tipo de entidad de educación superior que elegirán los estudiantes egresados, utilizamos bases de datos de registro de admisión de la Universidad de Concepción y del Instituto Profesional Virginio Gómez (IPVG), ambos de sus sedes de Chillán, y correspondientes a los años 2018 y 2019. Elegimos la región de Ñuble por ser la más reciente de Chile en cuanto a tiempo de existencia; y, en consecuencia, propicia para una investigación en esta línea.

La decisión de analizar esta investigación con los dos establecimientos antes mencionados obedece a una restricción de limitación de datos, junto con el hecho de que ambos pertenecen a una misma corporación y evidencian una alta diversidad de estudiantes en cuanto a sus situaciones socioeconómicas de origen (Universidad de Concepción, 2019). La cantidad de observaciones de los registros de admisión equivale a 2055 alumnos.

Desde un punto de vista econométrico, estimamos los factores que inciden en la probabilidad de que un estudiante haya continuado sus estudios en una universidad (UNIV) en vez de un instituto profesional (IP). Utilizamos un modelo Probit binario, puesto que la variable dependiente puede adoptar dos valores discretos (cero o la unidad).

Empleamos el Indicador de Desarrollo Personal y Social determinado por la Agencia de Calidad del Ministerio de Educación (2018) para la medición del grado de autoestima académica y motivación escolar (Unidad de Currículum y Evaluación del Ministerio de Educación, 2017). Además, incorporamos variables de control tales como características personales del estudiante y características del establecimiento de enseñanza media. La especificación del modelo econométrico es

$P\left(U N I V_{i}=1\right)=\phi\left(\beta_{0}+\beta_{1} A M_{i}+\Gamma X_{i}+\Delta W_{i}\right)+\varepsilon_{i}$

donde $U N I V_{i}$ es una variable dummy que toma valor $1 \mathrm{si}$ el i-ésimo estudiante accedió a la universidad para continuar sus estudios superiores, y 0 si accedió a un instituto. $A M$ corresponde al indicador de autoestima y motivación del establecimiento de origen, medido en logaritmo natural; $X_{i}$ es un vector de características del estudiante, mientras que $W_{i}$ es un vector de características del establecimiento de enseñanza media de egreso de este. Por otra parte, $P(\cdot)$ corresponde a una probabilidad de ocurrencia, $\phi(\cdot)$ es la función de probabilidad normal acumulada, $\beta_{0}$ y $\beta_{1}$ son parámetros por estimar, mientras que $\Gamma$ y $\Delta$ son vectores de parámetros. Finalmente, $\varepsilon_{i}$ representa el error de estimación.

El detalle de las variables empleadas y su descripción se encuentra en la Tabla 1.
Tabla 1. Descripción de variables

\begin{tabular}{|c|c|}
\hline Variable & Descripción \\
\hline \multicolumn{2}{|l|}{ Dependiente } \\
\hline Institución & $\begin{array}{l}\text { Dummy que toma valor } 1 \text { si el(la) estudiante fue } \\
\text { matriculado(a) en la Universidad de Concepción, } \\
\text { y } 0 \text { en el IPVG. }\end{array}$ \\
\hline \multicolumn{2}{|l|}{ Independientes } \\
\hline $\mathrm{AM}$ & $\begin{array}{l}\text { Indicador que mide el grado de autoestima y motivación } \\
\text { del establecimiento de enseñanza media de egreso del } \\
\text { (la) estudiante del año } 2016 \text {, medido en logaritmo natural. }\end{array}$ \\
\hline $\begin{array}{l}\text { Particular } \\
\text { subvencionado }\end{array}$ & $\begin{array}{l}\text { Dummy que toma valor } 1 \text { si el (la) estudiante egresó } \\
\text { de un establecimiento particular subvencionado, } \\
\text { y } 0 \text { en otro caso. }\end{array}$ \\
\hline $\begin{array}{l}\text { Particular } \\
\text { pagado }\end{array}$ & $\begin{array}{l}\text { Dummy que toma valor } 1 \text { si el (la) estudiante egresó de } \\
\text { un establecimiento particular pagado, y } 0 \text { en otro caso. }\end{array}$ \\
\hline Municipal & $\begin{array}{l}\text { Dummy que toma valor } 1 \text { si el (la) estudiante egresó } \\
\text { de un establecimiento municipal, y } 0 \text { en otro caso. }\end{array}$ \\
\hline Sexo & $\begin{array}{l}\text { Dummy que toma valor } 1 \text { si el estudiante es de sexo } \\
\text { masculino, y } 0 \text { en otro caso. }\end{array}$ \\
\hline Región Ñuble & $\begin{array}{l}\text { Dummy que toma valor } 1 \text { si el establecimiento de egreso } \\
\text { del estudiante se ubica dentro de la región de Nuble, } \\
\text { y } 0 \text { en otro caso. }\end{array}$ \\
\hline $\begin{array}{l}\text { Conurbación } \\
\text { Chillán }\end{array}$ & $\begin{array}{l}\text { Dummy que toma valor } 1 \text { si el establecimiento de egreso } \\
\text { del estudiante se ubica dentro de las comunas de Chillán } \\
\text { y Chillán Viejo, y } 0 \text { en otro caso. }\end{array}$ \\
\hline
\end{tabular}

Fuente: elaboración propia.

\section{Resultados}

Las cifras asociadas a la estadística descriptiva de las variables se encuentran detalladas en la Tabla 2.

Tabla 2. Estadística descriptiva

\begin{tabular}{lccccc}
\hline \multicolumn{1}{c}{ Variable } & Obs. & Media & D.E. & Mín & Máx \\
\hline Institución & 2055 & 0.420 & 0.494 & 0 & 1 \\
AM & 2049 & 4.319 & 0.034 & 4.174 & 4.443 \\
Particular subvencionado & 2055 & 0.525 & 0.499 & 0 & 1 \\
Particular pagado & 2055 & 0.139 & 0.346 & 0 & 1 \\
Municipal & 2055 & 0.335 & 0.472 & 0 & 1 \\
Sexo & 2055 & 0.420 & 0.494 & 0 & 1 \\
Región Nouble & 2055 & 0.786 & 0.410 & 0 & 1 \\
Conurbación Chillán & 2055 & 0.531 & 0.499 & 0 & 1 \\
\hline
\end{tabular}

Fuente: elaboración propia.

Podemos observar que casi la mitad de los estudiantes de la muestra accedió a la Universidad de Concepción, y el resto al IPVG. Gran parte de los establecimientos de enseñanza media de nuestra muestra presenta indicadores de autoestima y motivación bastante altos. Por otro lado, más de la mitad de estos son particulares subvencionados, el 33.5\% de ellos son de administración municipal, y casi el $14 \%$ son particulares pagados. El $42 \%$ de los estudiantes de la muestra es de sexo masculino. Además, casi el $80 \%$ de los 
estudiantes egresó de establecimientos pertenecientes a la región de Ñuble, mientras que el 49.9 \% son de las comunas de Chillán y Chillán Viejo.

Los resultados econométricos de nuestras estimaciones Probit se encuentran detallados en la Tabla 3. De manera preliminar, apreciamos que las cuatro especificaciones son significativas de acuerdo con la prueba de Wald; y, además, se estimaron con errores estándares robustos para solucionar potenciales problemas de heterocedasticidad. Respecto a la pregunta de investigación, los efectos marginales de la variable que mide el grado de autoestima y motivación son todos positivos y significativos en la mitad de las estimaciones. Esto quiere decir que si un estudiante egresa de un establecimiento de enseñanza media con un mayor grado de autoestima y motivación, aumenta la probabilidad de que entre a la Universidad de Concepción en vez del IPVG. Esta evidencia es coherente con nuestra hipótesis de investigación.

En cuanto a las variables de control, los resultados muestran que, por un lado, la probabilidad de que un estudiante entre a la universidad en vez de a un instituto profesional se incrementa si su establecimiento de egreso es particular subvencionado, y se reduce si este es municipal o particular pagado. Por otro lado, dicha probabilidad aumenta si el estudiante es de sexo masculino y se reduce si el alumno estudió en un establecimiento perteneciente a la región de Nuble o a la conurbación Chillán - Chillán Viejo (versus alumnos que provienen de otras regiones y ciudades), que son las localidades a las cuales pertenecen las dos entidades de educación superior tratadas en este estudio.

En síntesis, dado que los establecimientos de enseñanza media influyen sobre el grado de autoestima y motivación de sus estudiantes en cuanto a sus resultados académicos, este sería un factor relevante en cuanto a la posibilidad que estos tendrán al momento de postular a instituciones de educación superior. La probabilidad de entrar a una universidad se incrementa ante una mayor influencia por parte del establecimiento en este aspecto del desarrollo personal y social de los alumnos. Nuestra investigación presenta estas implicancias para los casos de la Universidad de Concepción y el Instituto Profesional Virginio Gómez, ambos para sus sedes de la comuna de Chillán.

\section{Conclusiones}

En Chile, el sistema de educación superior ha avanzado en cobertura, en acceso, en reorganizaciones ministeriales, en descentralizaciones y en distintos programas de subvención, pero el sistema también avanzar en desarrollar programas en congruencia con la realidad de cada establecimiento educacional que permitan construir
Tabla 3. Regresiones probit, efectos marginales

\begin{tabular}{|c|c|c|c|c|}
\hline Variable & (1) & (2) & (3) & (4) \\
\hline \multirow{2}{*}{$\mathrm{AM}$} & $0.491 *$ & 0.150 & $0.755^{* *}$ & 0.419 \\
\hline & $(0.296)$ & $(0.308)$ & $(0.309)$ & $(0.319)$ \\
\hline \multirow{2}{*}{$\begin{array}{l}\text { Particular } \\
\text { subvencionado }\end{array}$} & $0.118^{* * *}$ & $0.151 * * *$ & & \\
\hline & $(0.0230)$ & $(0.0250)$ & & \\
\hline \multirow{2}{*}{$\begin{array}{l}\text { Particular } \\
\text { pagado }\end{array}$} & $-0.305^{* * *}$ & $-0.286 * * *$ & & \\
\hline & $(0.0263)$ & $(0.0326)$ & & \\
\hline \multirow{2}{*}{ Municipal } & & & $-0.0433 *$ & $-0.0891 * * *$ \\
\hline & & & $(0.0228)$ & $(0.0250)$ \\
\hline \multirow{2}{*}{ Sexo } & $0.0644 * * *$ & $0.0711^{* * *}$ & $0.0703 * * *$ & $0.0776 * * *$ \\
\hline & $(0.0205)$ & $(0.0211)$ & $(0.0215)$ & $(0.0220)$ \\
\hline \multirow{2}{*}{ Región Ñuble } & $-0.291 * * *$ & & $-0.322 * * *$ & \\
\hline & $(0.0255)$ & & $(0.0254)$ & \\
\hline \multirow{2}{*}{$\begin{array}{l}\text { Conurbación } \\
\text { Chillán }\end{array}$} & & $-0.0824 * * *$ & & $-0.130 * * *$ \\
\hline & & $(0.0235)$ & & $(0.0234)$ \\
\hline Observaciones & 2049 & 2049 & 2049 & 2049 \\
\hline Dummy tiempo & Sí & Sí & Sí & Sí \\
\hline Wald $X^{2}$ & $(331.97)^{* * *}$ & $(208.67)^{* * *}$ & $(155.00)^{* * *}$ & $(46.07)^{* * *}$ \\
\hline Pseudo $R^{2}$ & 0.1198 & 0.0769 & 0.0581 & 0.0174 \\
\hline
\end{tabular}

Notas: errores estándares robustos en paréntesis. Los superíndices ***,** y * indican significancia estadística al 1,5 y $10 \%$, respectivamente. Fuente: elaboración propia.

políticas públicas dirigidas a potenciar las competencias de sus educandos, específicamente su formación actitudinal, esto es, la autoestima y la motivación. En este sentido, es indispensable propiciar estos factores para que cada estudiante desarrolle de forma exitosa su proyecto de vida deseado, en relación con lo planteado por Castañeda (2011), más allá de que se encuentre condicionado a un tipo de entidad de educación superior.

Sobre la base de los resultados de este estudio, se ha encontrado evidencia de que los estudiantes que egresan de establecimientos educacionales secundarios con mayor autoestima y motivación, medidos por la Agencia de Calidad del Ministerio de Educación, tienen mayor probabilidad de ingresar a la Universidad de Concepción que al Instituto Profesional Virginio Gómez.

Bajo esta perspectiva, resulta conveniente ampliar la línea de investigación en dos enfoques. Primero, extender el análisis cuantitativo a una muestra nacional de universidades e institutos. Segundo, complementar dicho análisis a través de estudios cualitativos, según lo citado por Irvine (2018), que permitan conocer la manera en que cada establecimiento secundario incluye la autoestima y motivación, no solo académica y escolar, en sus métodos de enseñanza-aprendizaje. En este sentido, al considerar la autoestima y la motivación dirigidas hacia un punto de vista de logro académico, que se condice con motivaciones extrínsecas (Elliot y McGregor, 2001), se limitaría el 
análisis de estos factores desde la perspectiva de que cada estudiante pueda concretar su proyecto de vida deseado.

Lo anterior genera un marco de conversación, considerando a D'Angelo (1986), acerca de cuál es la forma en que los agentes de una comunidad escolar deberían orientar la motivación y la autoestima en todas sus dimensiones, con lo cual se abordarían aspectos cualitativos para próximas investigaciones que orienten la formación de políticas públicas que guíen a los estudiantes secundarios en la decisión de ingreso al establecimiento de educación superior coincidente con su proyecto de vida.

\section{Referencias}

Abouserie, R. (1995). Self-esteem and achievement motivation as determinants of students' approaches to studying. Studies in Higher Education, 20(1), 19-26.

Arias, E., Farías, M., González, C., Huneeus, C., \& Rucci, G. (2015). Educación técnico profesional en Chile. Banco Interamericano de Desarrollo.

Arnold, D., \& Doctoroff, G. (2003). The early education of socioeconomically disadvantaged children. Annual review of psychology, 54(1), 517-545.

Arón, A. \& Milicic, N. (2004). Clima Social Escolar y Desarrollo Personal. Andrés Bello.

Awan, R., Noureen, G., \& Naz, A. (2011). A Study of Relationship between Achievement Motivation, Self-Concept and Achievement in English and Mathematics at Secondary Level. International Education Studies, 4(3), 72-79.

Aziz, C. (2018). Evolución e implementación de las políticas educativas en Chile. Nota técnica $N^{o} 2$. Líderes educativos, Centro de Liderazgo para la Mejora Escolar: Chile.

Barker, R., McInerney, D. \& Dowson, M. (2003). The Effects of Motivational Conditions on the Mathematics Performance of Students on the National Assessment of Educational Progress Assessment, Yayinlanmamiş Doktora Tezi. Georgia State University.

Bénabou, R., \& Tirole, J. (2003). Intrinsic and extrinsic motivation. Review of Economic Studies, 70, 489-520.

Blanco, R., \& Cusato, S. (2004). Desigualdades educativas en América Latina: todos somos responsables. Escuelas de Calidad en condiciones de pobreza. http://dcsh.xoc.uam.mx/planeacion/bibliografia2014/ Desigualdades_BLANCO.pdf

Busso, M., Bassi, M., Urzúa, S., \& Vargas, J. (2012). Desconectados: habilidades, educación y empleo en América Latina. Inter-American Development Bank.
Castañeda, L. (2011). Un plan de vida para jóvenes: ¿Qué harás con el resto de tu vida? Panorama.

Conley, A., \& Karabenick, S. (2006). Construct validity issues in the measurement of motivation to learn. En Biennial meeting of the Society for Research on Adolescence. https://citeseerx.ist.psu.edu/viewdoc/download?doi=10.1.1.586.317\&rep=rep1\&type $=$ pdf

D’Angelo, O. (1986). La formación de los proyectos de vida del individuo: Una necesidad social. Revista cubana de psicología, 3(2), 31-39.

Elliot, A., \& McGregor, H. (2001). A $2 \times 2$ achievement goal framework. Journal of personality and social psychology, 80(3), 501.

García, F., \& Musitu, G. (1993). Un programa de intervención basado en la autoestima: análisis de una experiencia. Revista de Psicología Universitas Tarraconensis, 15(1), 57-78.

Green, H. (2012). From paternalism to participation: the motivations and understandings of the "developers". Development in Practice, 22(8), 1109-1121.

Himmel, E. (2002). Modelos de análisis de la deserción estudiantil en la educación superior. Revista Calidad en la educación, 17(2), 91-108.

Irvine, J. (2018). A Framework for Comparing Theories Related to Motivation in Education. Research in Higher Education Journal, 35.

Llanos, E., Medina, V., Ortiz, M., Karmach, E. \& Herrera, R. (2018). Análisis de los perfiles de ingreso y su relación con el rendimiento académico. Multidisciplinary Business Review, 11(1), 13-26.

Lomelí, A., López, M. \& Valenzuela, J. (2016). Autoestima, motivación e inteligencia emocional: Tres factores influyentes en el diseño exitoso de un proyecto de vida de jóvenes estudiantes de educación media. Revista Electrónica Educare, 20 (2), 1-22.

Mujis, D. \& Reynold, D. (2005). Effective teaching, evidence and practice. Sage.

OCDE. (2015). In it Together. En In it Together: Why Less Inequality Benefits All (pp. 1-336). OCDE Publishing.

Rodríguez, E. (2012). La educación superior en Chile y el rol del mercado: ¿culpable o inocente? Ingeniare. Revista chilena de ingeniería, 20(1), 126-135.

Ryan, R., \& Deci, E. (2000). Intrinsic and Extrinsic Motivations: Classic Definitions and New Directions. Contemporary Educational Psychology, 25(1), 54-67.

Sepúlveda, L. \& Valdebenito M., (2014) ¿Las cosas claras? Aspiraciones de futuro y proyecto educativo laboral de jóvenes estudiantes secundarios. Estudios pedagógicos, 40(1), 243-261. 


\section{Fuentes electrónicas}

Agencia de Calidad de la Educación (2018). Informe técnico 2017, Indicadores de Desarrollo Personal y Social (IDPS). Ministerio de Educación de Chile. https://bibliotecadigital.mineduc.cl/bitstream/ handle/20.500.12365/4572/IDPS_2017.pdf?sequence $=1 \&$ isAllowed $=\mathrm{y}$

Comisión Nacional de Acreditación (2019). Análisis del aseguramiento de la calidad de la educación superior. Barómetro Del Aseguramiento de La Calidad de La Educación Superior ( $\left.N^{\circ} 4\right)$. http://www.cnachile.cl/

Sánchez, I. (2011). Los desafios de la educación superior en Chile. Centro de Estudios Públicos UC. https:// rectoria.uc.cl/images/stories/docs/desafios_de_la_educacion_superior.pdf
Unesco. (2010 - 2011). World data on education. Datos mundiales de educación ( $7^{\circ}$ ed.). http:// www.ibe.unesco.org/es/documento/datos-mundiales-de-educaci\%C3\%B3n-s\%C3\%A 9ptima-edici\%C3\%B3n-2010-11

Unidad de Currículum y Evaluación Ministerio de Educación (2017). Autoestima académica y motivación escolar. https://www.curriculumnacional.cl/614/articles-90116_recurso_2.pdf

Universidad de Concepción (2019). Corporación Universidad de Concepción. Presentación Corporativa- octubre 2019. http://www.udec.cl/pexterno/sites/default/ files/UDEC_NDR_vf_16_10_2019.pdf 